\title{
Photoactivatable RNAi for cancer gene therapy triggered by near-infrared-irradiated single-walled carbon nanotubes
}

This article was published in the following Dove Press journal:

International Journal of Nanomedicine

26 October 2017

Number of times this article has been viewed

\author{
Xueling Ren \\ Jing Lin \\ Xuefang Wang \\ Xiao Liu \\ Erjuan Meng \\ Rui Zhang \\ Yanxiao Sang \\ Zhenzhong Zhang
}

Key Laboratory of Targeting Therapy and Diagnosis for Critical Diseases, School of Pharmaceutical Sciences, Zhengzhou University, Zhengzhou, People's Republic of China
Correspondence: Zhenzhong Zhang Key Laboratory of Targeting Therapy and Diagnosis for Critical Diseases, School of Pharmaceutical Sciences, Zhengzhou University, 100 Kexue Avenue, Zhengzhou, Henan, People's Republic of China $\mathrm{Tel}+8637 \mid 67781910$ Fax +86 37| 6778 I908 Email zhenzhongz@I26.com

\begin{abstract}
The efficacy of RNA interference (RNAi)-based cancer gene therapy is limited by its unexpected side effects, thus necessitating a strategy to precisely trigger conditional gene knockdown. In this study, we engineered a novel photoactivatable RNAi system, named as polyetherimide-modified single-wall carbon nanotube (PEI-SWNT)/pHSP-shT, that enables optogenetic control of targeted gene suppression in tumor cells. PEI-SWNT/pHSP-shT comprises a stimulus-responsive nanocarrier (PEI-SWNT), and an Hsp70B'-promoter-driven RNAi vector (pHSP-shT). In response to near-infrared (NIR) light irradiation, heating of PEI-SWNT in breast MCF-7 cells triggered gene knockdown targeting human telomerase reverse transcriptase through RNAi, with the gene-knockdown activity capable of being switched off by extinguishing the NIR. Furthermore, we demonstrated that the photoactivatable RNAi system exhibited higher antitumor activity by combining gene therapy and photothermal therapy, both in vitro and in vivo. Optogenetic control of RNAi based on an NIR-activated nanocarrier will potentially facilitate improved understanding of molecular-targeted gene therapy in human malignant tumors.
\end{abstract}

Keywords: near-infrared light response, SWNT, RNAi, Hsp70B' promoter

\section{Introduction}

RNA interference (RNAi) is one of the most common techniques used in gene therapy of malignant tumors. ${ }^{1}$ RNAi can result in post-transcriptional gene silencing of endogenous target genes. However, the potential unexpected side effects of exogenous gene therapy resulting from inexplicit regulation and control should not be neglected, ${ }^{2}$ because gene expression is rigorously controlled by cells to maintain precise internal homeostasis of normal human tissues, as well as tumors. Therefore, enhancing the effectiveness of RNAi-based gene therapy by precisely regulating conditional gene knockdown to reduce the level of off-target effects is an urgent concern.

The stimuli-responsive nanoparticle drug delivery system is a novel smart tool that can produce chemical or physical changes in response to external stimulation to implement targeted drug release. ${ }^{3}$ Near-infrared (NIR) light is a common type of energy with numerous merits, including easy access, environmental friendliness, and moderate and remotely controllable properties, that allow it to be widely used for establishing stimulus-responsive biomaterials. ${ }^{4}$ Studies have revealed that the controlled release of drugs by alternating the presence and absence of NIR can effectively increase drug concentrations in target areas and simultaneously minimize the toxicity caused by premature release during distribution. ${ }^{5}$ These nanoparticle drug delivery systems have been used for controlled release of small chemical drugs and small-interfering 
RNA, ${ }^{6}$ and their success has encouraged meaningful exploration of novel NIR-responsive gene delivery systems that could facilitate both management of exogenous gene expression and achieving targeted and controllable tumor therapy treatment.

An essential problem in establishing a smart, stimulusresponsive, gene delivery system is the effective linking of nanoparticle responses to exogenous gene expression. A previous study reported that cells can overexpress heat-shock protein $70 \mathrm{~B}^{\prime}$ (Hsp70B') under heat or hypoxic conditions, ${ }^{7}$ and that the increase in expression represents a transcriptional regulatory mechanism. ${ }^{8}$ Therefore, the Hsp70B' promoter has been one of the most commonly used promoters for regulating timing and targeting gene expression to enable targeted gene therapy for tumors. An Hsp70B'-promoterdriven vector has been used to photothermally express a cytokine in B16 melanoma tumors. ${ }^{9}$ Therefore, in this study, we set out to design a novel NIR-responsive RNAi system based on the Hsp70B' promoter that enables photothermal control of RNAi efficiency in malignant tumor cells, and to evaluate the antitumor activity of the controllable gene therapy system both in vitro and in vivo.

\section{Experimental procedures Materials}

The single-wall carbon nanotube (SWNT) was purchased from the Chinese Academy of Sciences Chengdu Institute of Organic Chemistry (Chengdu, People's Republic of China). $\mathrm{T}_{4}$ DNA ligase, Taq DNA polymerase, and Pfu DNA polymerase were purchased from Promega (Durham, NC, USA). The restriction endonucleases NheI, VspI, EcoRI, and XbaI were purchased from Fermentas (Waltham, MA, USA). Dulbecco's Modified Eagle's Medium (DMEM) and fetal bovine serum (FBS) were purchased from Hyclone (GE Life Sciences, Pittsburgh, PA, USA), and all chemicals were purchased from Amresco (Solon, OH, USA). Primer synthesis and DNA sequencing were conducted by the Beijing Sanboyuanzhi Biotech Corporation (Beijing, People's Republic of China).

\section{Cell lines and cell proliferation}

The human breast cancer cell line MCF-7 was obtained from the Cell Bank of the Chinese Academy of Science (Shanghai, People's Republic of China) and cultured in DMEM with $10 \% \mathrm{FBS}$ at $37^{\circ} \mathrm{C}$ and $5 \% \mathrm{CO}_{2}$ in a humidified incubator (MCO-15AC; Sanyo, Osaka, Japan). The cell proliferation rate was measured by using the 3-(4,5-dimethyl-2-thiazolyl)2,5-diphenyl-2-H-tetrazolium bromide (MTT) assay.

\section{Plasmid construction}

pCMV-GFP was a green fluorescent protein (GFP) expression vector containing a cytomegalovirus (CMV) promoter. To establish a heat-inducible GFP vector pHSP-GFP, the Hsp70B' promoter was amplified by polymerase chain reaction (PCR) from the genome of MCF-7 cells and inserted into the $V s p \mathrm{I} /$ NheI site of pCMV-GFP to replace the CMV promoter. The pCMV-shGFP vector was an RNAi vector targeting GFP. To construct pHSP-shGFP - a heat-inducible RNAi vector - the CMV promoter of pCMV-shGFP was replaced with the $\mathrm{Hsp} 70 \mathrm{~B}^{\prime}$ promoter. The primers used to amplify the Hsp70B' promoter were 5'-GGC ATT AAT AGC CCG GAG GAG CTA GAA CCT TCC-3' (upstream) and 5'-CCC GCT AGC TTC TTG TCG GAT GCT GGA GGC-3' (downstream).

To construct pHSP-shT - a heat-inducible RNAi vector targeting human telomerase reverse transcriptase (hTERT) two DNA oligonucleotides containing the hTERT-targeting sequence were chemically synthesized and annealed to form a DNA fragment, and the DNA oligonucleotide chain was inserted into the $E c o$ RI/XbaI site of pHSP-shGFP (Figure 1). The hTERT target sequence was 5'-TTC CGC CAG GTG TCC TGC CTG AAG GAG CT-3'. All plasmids were verified by sequencing.

\section{Assessment of the transfection and transcription activity of the Hsp70 ${ }^{\prime}$ promoter}

MCF-7 cells were seeded in a 96 -well plate $\left(1.2 \times 10^{4}\right.$ cells $/$ well $)$. Upon reaching $\sim 70 \%$ confluence, $\mathrm{pHSP}-\mathrm{GFP}$ was transfected into the cells using the transfection reagent ExGen500

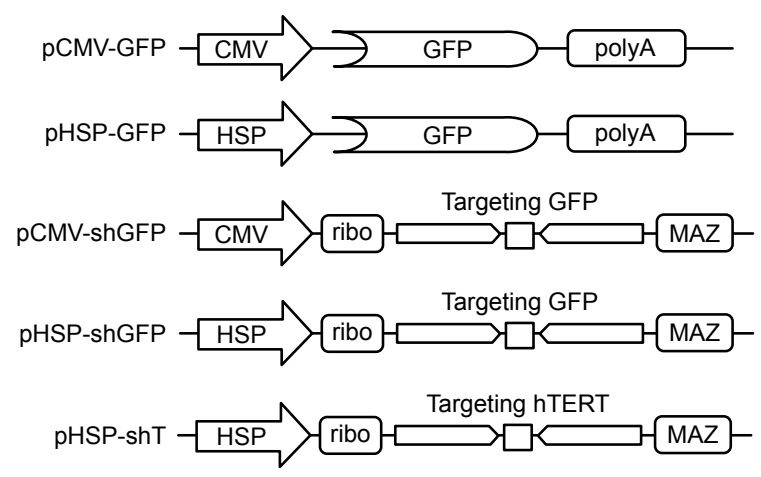

Figure I Schematic representation of plasmids used in this work. PCMV-GFP and PHSP-GFP are GFP expression plasmids with different promoters (CMV or Hsp70B', respectively). pCMV-shGFP is a CMV-promoter-driven short hairpin RNA (shRNA) expression construct consisting of the cis-acting hammerhead ribozyme (ribo) immediately downstream of the CMV promoter, a gene-specific shRNA cassette targeting GFP, and a specific pausing site MAZ. PHSP-shGFP and pHSP-shT, structurally similar to PCMV-shGFP, are heat-inducible RNAi vectors containing the Hsp70B' promoter.

Abbreviations: GFP, green fluorescent protein; CMV, cytomegalovirus; HSP, heatshock protein; sh, short hairpin. 
(Biomol GmbH, Hamburg, Germany) according to the manufacturer's protocol. Briefly, $0.3 \mu \mathrm{g}$ plasmid was complexed with ExGen500 in $150 \mathrm{mM} \mathrm{NaCl}$ and transferred into each well. After $24 \mathrm{~h}$, the transfected cells were incubated at $40^{\circ} \mathrm{C}$, $42^{\circ} \mathrm{C}$, or $44^{\circ} \mathrm{C}$ for 20,30 , or $40 \mathrm{~min}$, respectively. At $48 \mathrm{~h}$ after heat induction, GFP expression was determined by flow cytometry (BD Biosciences, San Diego, CA, USA).

\section{Synthesis and characterization of polyetherimide-modified single-wall carbon nanotube (PEI-SWNT)}

PEI-SWNT was prepared according to our previous work. ${ }^{10}$ First, carboxylic SWNT (SWNT-COOH) was synthesized using concentrated sulfuric acid, concentrated nitric acid, and hydrogen peroxide to oxidize the SWNT. Ethylenediamine was then introduced to SWNT-COOH by a condensation reaction between carboxyl and amidogen to form SWNT$\mathrm{NH}_{2}$. Eventually, PEI-SWNT was obtained following a polymerization reaction between aziridine and amidogen. PEI-SWNT was characterized by Fourier transform infrared (FTIR; IR-408; Shimadzu, Kyoto, Japan) spectroscopy and thermal gravimetric analysis (TGA) using a thermogravimetric analyzer (TGA-50; Shimadzu) under the following conditions: scanning from $20^{\circ} \mathrm{C}$ to $800^{\circ} \mathrm{C}$, a nitrogen flow rate of $40 \mathrm{~mL} / \mathrm{min}$, and a heating rate of $10^{\circ} \mathrm{C} / \mathrm{min}$.

\section{Preparation and characterization of PEI-SWNT/PCMV-GFP nanoparticles}

PEI-SWNT and pCMV-GFP were mixed at mass ratios of $0: 1,2: 1,4: 1,6: 1$, and $8: 1$ and kept at room temperature for $30 \mathrm{~min}$ to form PEI-SWNT/pCMV-GFP nanoparticles. The loading effect was detected via $0.7 \%$ agarose gel electrophoresis, and size distribution and the zeta potentials of the nanoparticles were measured with a laser particle-size analyzer (Zetasizer Nano ZS-90; Malvern, Malvern, UK). The ultraviolet-visible light (UV-Vis) spectrogram of the nanoparticle was measured by using a spectrophotometer (UV-3600; Shimadzu).

The PEI-SWNT/pCMV-GFP nanoparticle was transfected into MCF-7 cells and, after $48 \mathrm{~h}$, the cells were digested and examined by flow cytometry (BD Biosciences) to determine GFP expression.

\section{Activation of Hsp70B' promoter transcription by NIR in vitro}

At $16 \mathrm{~h}$ before transfection, MCF-7 cells were plated onto six-well plates $\left(5 \times 10^{5}\right.$ cells/well), and PEI-SWNT/pHSPGFP nanoparticles at a mass ratio of 6:1 were transfected into MCF-7 cells at a dosage of $3 \mu \mathrm{g}$ DNA/well. After $24 \mathrm{~h}$ culture, cells were exposed to $808 \mathrm{~nm} \mathrm{NIR}$ at $1.5 \mathrm{~W} / \mathrm{cm}^{2}$ for $45 \mathrm{~s}$. Following $48 \mathrm{~h}$ culture, treated MCF-7 cells were imaged via fluorescence microscopy (Zeiss LSM510; Carl Zeiss, Oberkochem, Germany) to qualitatively visualize GFP expression. The transfection efficiency of PEI-SWNT with pCMV-GFP/pHSP-shGFP (mass ratio of pCMV-GFP/pHSPshGFP $=3: 1$ ) was determined using a similar protocol.

\section{Reverse transcription-PCR analysis}

Total RNA from transfected MCF-7 cells treated with radiation was extracted using TRIzol (Tiangen, Beijing, People's Republic of China). Reverse transcription (RT) was conducted using $2 \mu \mathrm{L}$ RNA, and specific primers were used for PCR amplification. The hTERT primers were 5'-CGG AAG AGT GTC TGG AGC AA-3' (upstream) and 5'-CAC GAC GTA GTC CAT GTT CA-3' (downstream), and the $\beta$-actin primers were $5^{\prime}$-CTG GGA CGA CAT GGA GAA AA-3' (upstream) and 5'-AAG GAA GGC TGG AAG AGT GC-3' (downstream). PCR products were separated via $1 \%$ agarose gel electrophoresis.

\section{Western blot}

MCF-7 cells were digested and lysed using a radioimmunoprecipitation assay lysis solution to extract the proteins in an ice bath. The protein concentration was determined via the BCA assay, and proteins were separated with sodium dodecyl sulfate polyacrylamide gel electrophoresis. Proteins were transferred to a membrane, and then the membrane was blocked with $5 \%$ nonfat dry milk and incubated with antibodies for hTERT $(1: 1,000)$ or $\beta$-actin $(1: 2,000)$ at $4{ }^{\circ} \mathrm{C}$ for $12 \mathrm{~h}$. After washing with Tris-buffered saline containing Tween-20, the blots were visualized via enhanced chemiluminescence and analyzed using Quantity One software (Bio-Rad, Hercules, CA, USA).

\section{Tumor model in nude mice and anti- tumor activity in vivo}

Female BALB/c nude mice (4-6 weeks old) were obtained from Beijing Weitonglida Laboratory Animals Corporation, Ltd (Beijing, People's Republic of China). Animal experiments were conducted according to the National Regulation on the Management of Laboratory Animals, and were approved by the Ethical Committee of Zhengzhou University. Tumor models were established by subcutaneous injection of MCF-7 cells $\left(2 \times 10^{6}\right.$ cells $)$ into the right shoulder of mice, and the mice were examined when tumor volume reached $60 \mathrm{~mm}^{3}$.

Mice harboring MCF-7 tumors were divided into four groups (six mice per group): the saline control group, 
pHSP-shT only group, PEI-SWNT nanocarrier group, and PEI-SWNT/pHSP-shT nanoparticle group. The doses of PEISWNT and pHSP-shT were 6 and $1 \mu \mathrm{g} / \mathrm{g}$, respectively, and mice were injected via their tail veins every 6 days. At $24 \mathrm{~h}$ post-injection, the tumor was exposed to NIR at $1.5 \mathrm{~W} / \mathrm{cm}^{2}$ for $1 \mathrm{~min}$. The mice were observed daily for clinical symptoms, and tumor volume and body weight were monitored every 3 days. At 21 days post-injection, the mice were sacrificed, and the major organs and tumor tissues were harvested. The tumor tissue and major organs, including the heart, liver, spleen, lung, and kidney, were weighed and used for hematoxylin and eosin (H\&E) staining, RT-PCR, and Western blot analysis.

\section{Statistical analysis}

All data are expressed as the mean \pm standard deviation, and data were analyzed for statistical significance using a $t$-test. $P<0.05$ was considered statistically significant.

\section{Results and discussion Cloning and heat-induced transcription efficiency of Hsp70B'}

Specific promoters enable targeted gene therapy for tumors by regulating the expression of therapeutic genes that can limit the expression of exogenous genes to targeted areas, thereby not only improving the therapeutic effect, but also eliminating the toxic effects of continuous expression of therapeutic genes in the peripheral area. Together with tissuespecific promoters, inducible promoters capable of being activated by external chemical or physical factors are widely used in gene therapy. ${ }^{11}$ In this study, we attempted to use a heat-inducible promoter to manipulate the RNAi effects of gene therapy for human breast cancer.

HSP70B', an important member of the HSP70 family, is dramatically upregulated in response to heat-shock conditions generated primarily by heat-induced transcription factors. As shown in Figure 2A, the Hsp70B' promoter contains a GATA box and lacks the typical TATA box and CAAT box sequences. Four conserved repeated sequences $(-103$ to $-94 \mathrm{bp},-93$ to $-84 \mathrm{bp},-83$ to $-74 \mathrm{bp}$, and -73 to $-64 \mathrm{bp}$ ) are characterized as heat-shock elements that direct the heat-induced transcription of the Hsp70B' promoter. ${ }^{12}$ Therefore, a fragment of $407 \mathrm{bp}(-288$ to $+119 \mathrm{bp})$ was used in this study.

To evaluate heat-induced transcription of the Hsp70B' promoter, the 407-bp fragment of the Hsp70B' promoter was cloned upstream of the GFP gene to create the pHSPGFP vector. GFP is a reporter gene frequently used for monitoring gene expression efficiency. ${ }^{13}$ The heat-induced plasmid pHSP-GFP and the control plasmid pCMV-GFP were transfected into MCF-7 cells in parallel. As expected, we observed extensive green fluorescence in pCMV-GFPtreated cells, whereas GFP expression was not detected in cells transfected with pHSP-GFP. When the pHSP-GFPtreated MCF-7 cells were heated, GFP expression increased dramatically in a temperature- and time-dependent manner (Figure 2B), suggesting that the Hsp70B' promoter regulated GFP expression under heat stimulation. Furthermore, we found that cell proliferation noticeably declined proportionally with rising temperature (Figure $2 \mathrm{C}$ ), indicating that heat inhibited cellular proliferation to some degree, in agreement with a previous report. ${ }^{14}$

We then focused on whether the Hsp70B' promoter could drive the heat-induced RNAi effect. Typically, RNAi expression vectors are driven by RNA polymerase III (Pol III) promoters such as U6, H7, and H1. However, the Hsp70B' promoter is a Pol II promoter. Because the transcript from the Pol II promoter has a 5'-cap and $3^{\prime}$-poly(A) structure owing to post-transcriptional modification, the limitation of the RNAi vector driven by the Hsp70B' promoter has off-target effects. ${ }^{15}$ In our previous work, we removed these unexpected segments using the cis-acting hammerhead ribozyme and MAZ-pausing site. ${ }^{16}$ Here, we constructed an Hsp70B' promoter-driven RNAi vector pHSP-shGFP targeting GFP by using a similar approach. To determine the RNAi regulation efficiency of the Hsp70B' promoter, the recombinant plasmid pHSP-shGFP was co-transfected with the GFP expression vector pCMV-GFP into MCF-7 cells at a ratio of $1: 1$. At $24 \mathrm{~h}$ post-transfection, the cells were incubated at $42^{\circ} \mathrm{C}$ for $40 \mathrm{~min}$ and cultured for an additional $48 \mathrm{~h}$. As shown in Figure 2D, GFP expression in heated cells was only $17.12 \% \pm 0.47 \%$, which was much lower than that of cells cultured without heat stimulation $(87.40 \% \pm 2.92 \%)$, indicating that the Hsp70B' promoter mediated heat-induced gene silencing against GFP.

\section{Construction and confirmation of the PEI-SWNT}

A prerequisite of stimulus-responsive gene therapy is that exogenous genes can be transfected and expressed in tumor cells. Although a few studies have reported that naked DNA directly injected into target regions can also express, most results have indicated that it is more effective to administer DNA using a well-designed drug delivery system. ${ }^{17,18}$ SWNT has been widely studied in biomedical research and plays a significant role in drug delivery systems. ${ }^{19,20}$ 

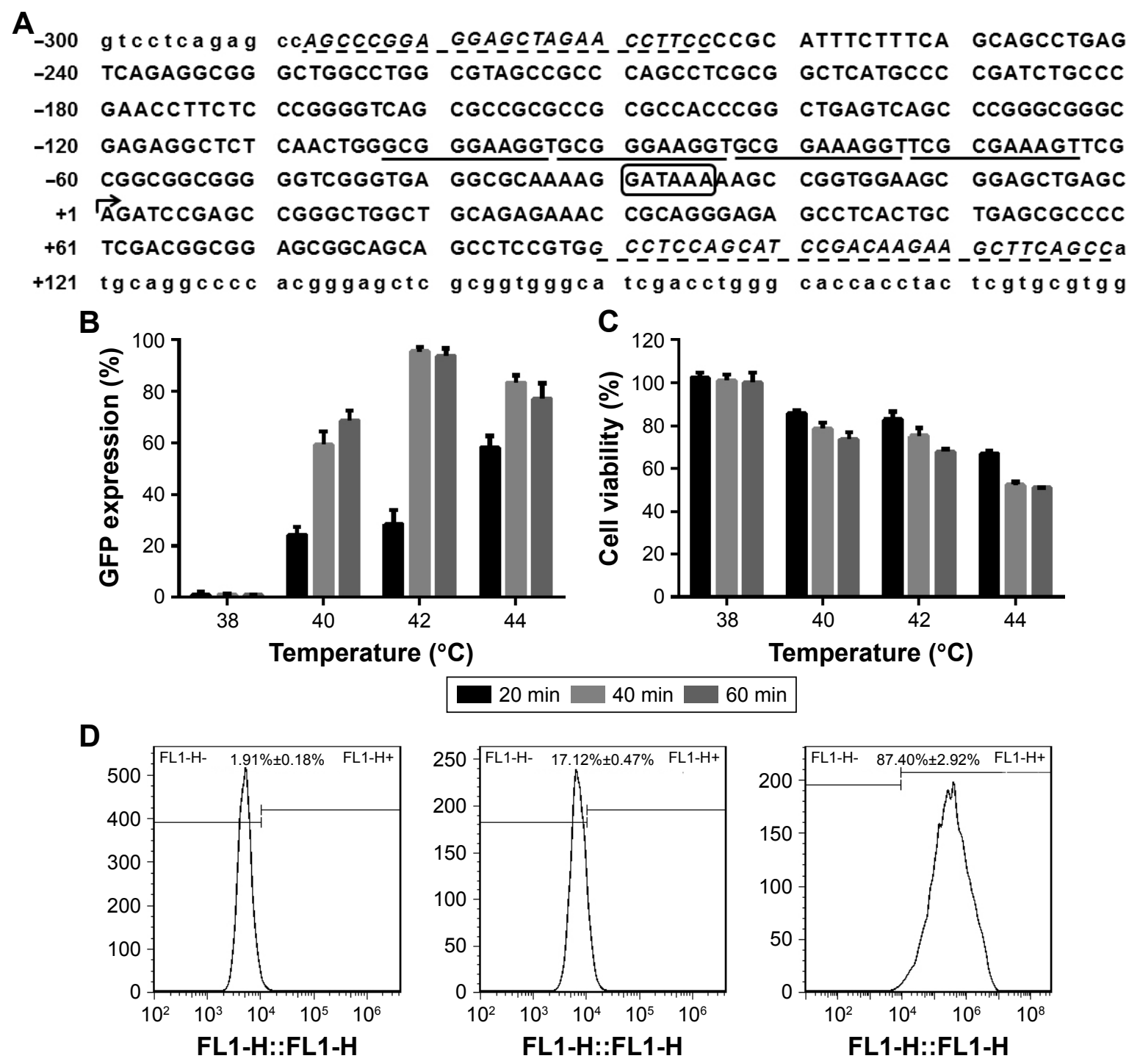

Figure 2 (A) The $5^{\prime}$-flanking sequence of the human Hsp70B' gene (GenBank accession: NT004487). The promoter sequence used in this work is shown in uppercase letters. Nucleotides matching the primers used for PCR amplification are in italics and indicated by dashed underlines. Four consecutive heat-shock-element sequences are underlined. The GATA box sequence is shown by a solid box. The transcription start site is represented by an arrow. (B) GFP expression and (C) cell viability of MCF-7 cells transfected with pHSP-GFP after heat-shock treatment at different temperatures and times. (D) Knockdown of GFP expression by pHSP-shGFP in MCF-7 cells. The indicated RNAi plasmid pHSP-shGFP was co-transfected into MCF-7 cells with the GFP expression plasmid pCMV-GFP at a ratio of I:I. At 24 h post-transfection, MCF-7 cells were treated with (middle) or without (right) heat-shock at $42^{\circ} \mathrm{C}$ for $40 \mathrm{~min}$, with untransfected MCF-7 cells used as controls (left).

Abbreviations: GFP, green fluorescent protein; HSP, heat-shock protein; PCR, polymerase chain reaction; sh, short hairpin.

Amino-functionalized SWNT and nucleic acid molecules can assemble a supramolecular complex by electrostatic interactions, forming a supramolecular complex that offers high degrees of transfection efficiency. Furthermore, SWNT can absorb NIR at 700-1,100 $\mathrm{nm}$ and convert luminous energy to thermal energy by an endogenous surface plasmon resonance effect. ${ }^{21}$ Therefore, we chose SWNT as a vector to deliver DNA and simultaneously induce gene expression.

As known, SWNT is both insoluble and aggregated rapidly in various aqueous solutions, and has poor capacity of nucleic acid loading due to its highly hydrophobic surface. To overcome these obstacles, SWNT was modified with highly positively charged soluble PEI by aziridine polymerization after carboxylation and amination of the SWNT. The resulting product showed excellent stability in PBS and serum conditions, and was characterized by FTIR and TGA. The FTIR spectrum of PEI-SWNT showed the presence of characteristic absorption peaks of amidogen at $1,632 \mathrm{~cm}^{-1}$ $\left(v_{-\mathrm{NH} 2}\right)$ and $1,446 \mathrm{~cm}^{-1}\left(\delta_{\mathrm{C}-\mathrm{N}}\right)$, which were lacking in SWNT (Figure 3A). TGA analysis further confirmed that PEI had been introduced at the surface of the SWNT. The difference between SWNT and PEI-SWNT is illustrated in Figure 3B. The results showed that the molecular weight of SWNT barely changed upon increases in environmental temperature, 


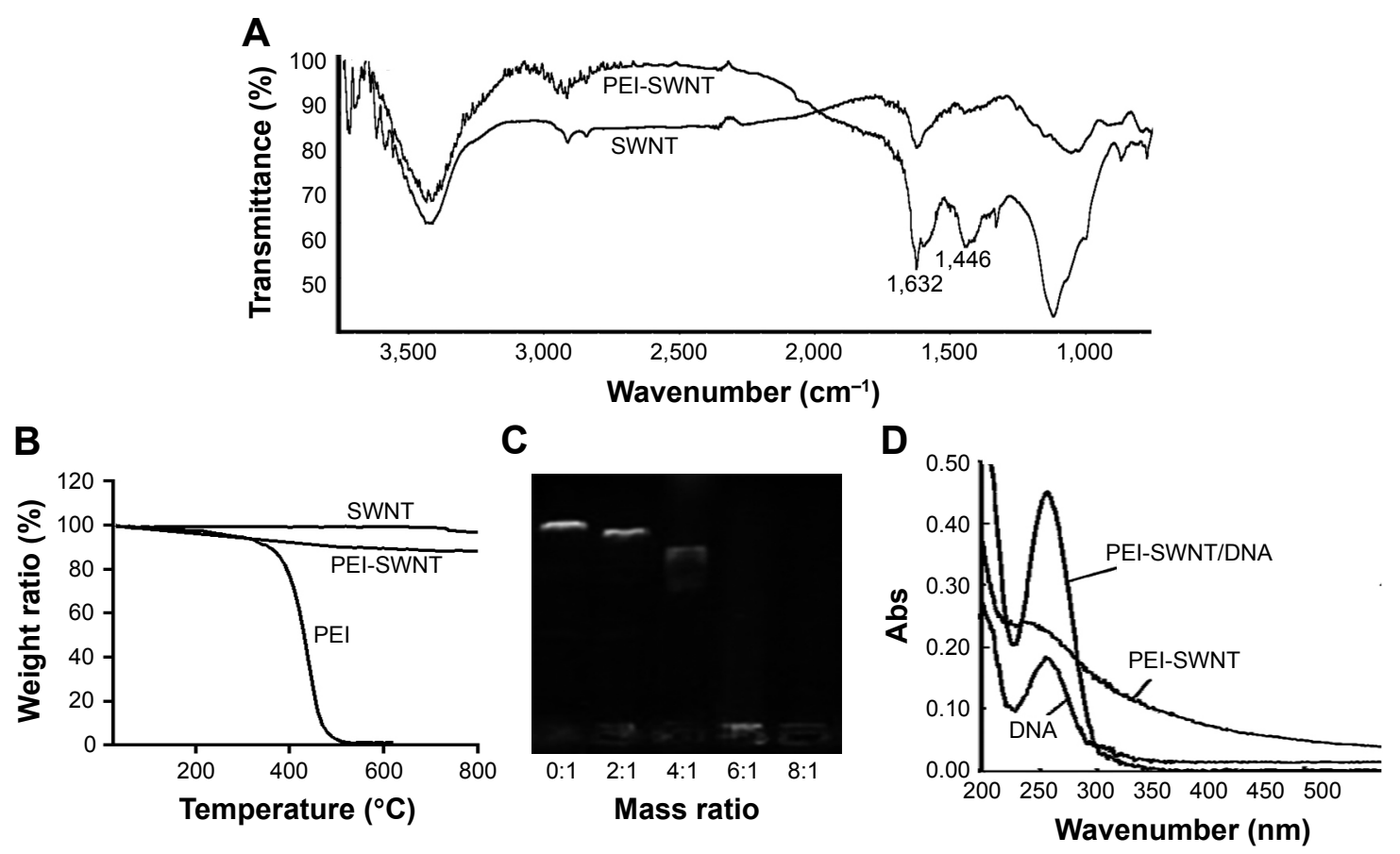

Figure 3 Characterization of PEI-SWNT by using (A) FTIR spectroscopy and (B) TGA. (C) Gel retardation of PEI-SWNT/DNA at different mass ratios. (D) UV-Vis spectrogram of PEI-SWNT/DNA.

Abbreviations: Abs, absorbance; FTIR, Fourier transform infrared; PEl, polyetherimide; PEI-SWNT, polyetherimide-modified single-wall carbon nanotube; SWNT, singlewall carbon nanotube; TGA, thermal gravimetric analysis; UV-Vis, ultraviolet-visible.

except for a slight drop above $500^{\circ} \mathrm{C}$, whereas the molecular weight of PEI-SWNT decreased at $\sim 300^{\circ} \mathrm{C}$, exhibiting relatively poor thermal stability because of the degradation of the PEI functional group at high temperatures. Both FTIR and TGA analysis confirmed the successful construction of PEI-SWNT.

Nucleic acids are negatively charged owing to their phosphate groups, enabling their migration toward positively charged electrodes under an electric field. When negatively charged nucleic acids are neutralized by positive charges, the migration rate is altered. Therefore, agarose gel electrophoresis constitutes an essential method for investigating the nucleic acid loading capability of vectors. As shown in Figure 3C, free nucleic acids were visualized as a single clear band in the gel. In the presence of PEI-SWNT, both the migration rate and luminance of nucleic acids declined, and the DNA band disappeared at PEI-SWNT:DNA mass ratios more than $6: 1$, indicating that the DNA was completely loaded by the PEI-SWNT.

The UV-Vis spectrum of the PEI-SWNT/DNA nanoparticle agreed with the results of gel electrophoresis, showing the presence of characteristic DNA peaks (Figure 3D). The size and zeta potential of a nanoparticle are important parameters for optimal drug delivery. Proper size promotes nanoparticle aggregation at the tumor area, and charges at the nanoparticle surface determine the dispersion stability, nucleic acid loading capacity, and ability to penetrate through the membrane. Size distribution and zeta potential were measured by laser particle-size analysis, which revealed that the mean size of free PEI-SWNT was $178.9 \mathrm{~nm}$, with a zeta potential of $+38.31 \mathrm{mV}$ (Figure S1). In contrast, the zeta potential of PEI-SWNT/DNA decreased to $8.07 \mathrm{mV}$ and its size was $\sim 200 \mathrm{~nm}$. These results confirmed DNA loading onto PEI-SWNT.

\section{Establishment and optimization of the NIR-triggered RNAi nano drug delivery system}

To determine the capability of PEI-SWNT to induce an NIR-triggered RNAi effect, we needed to ensure successful plasmid transfection into cells via PEI-SWNT, followed by verification that PEI-SWNT was capable of generating thermal energy via NIR to activate the Hsp70B' promoter.

Measurement of the transfection efficiency of PEISWNT following transfection of PEI-SWNT/pCMV-GFP nanoparticles into MCF-7 cells at mass ratios of 6:1 and $8: 1$, respectively, produced green fluorescence, whereas cells transfected with pCMV-GFP showed no fluorescence (Figure 4A), indicating that PEI-SWNT successfully transported the pCMV-GFP vector into cells.

Previously, we confirmed that the photothermal effect of PEI-SWNT induced by NIR occurred in a concentration- and 


\section{A pCMV-GFP}

\section{Without PEI-SWNT}

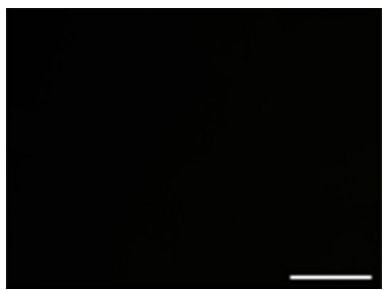

B PEI-SWNT/PHSP-GFP
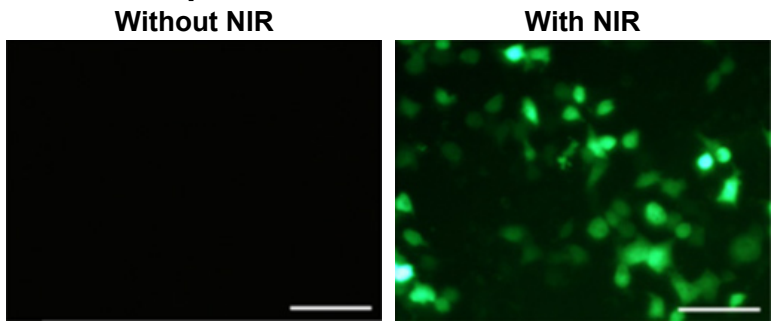

C PEI-SWNT/pCMV-GFP/pHSP-shGFP Without NIR

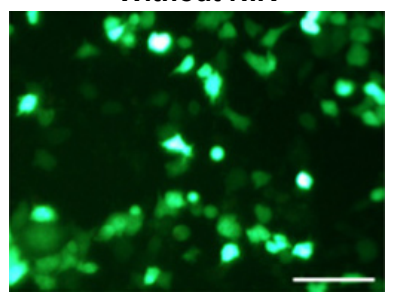

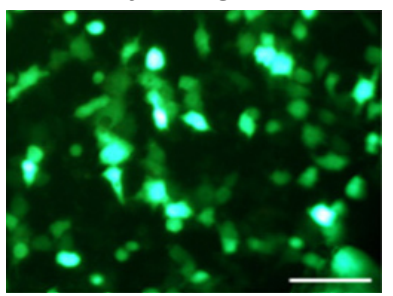

With PEI-SWNT

D

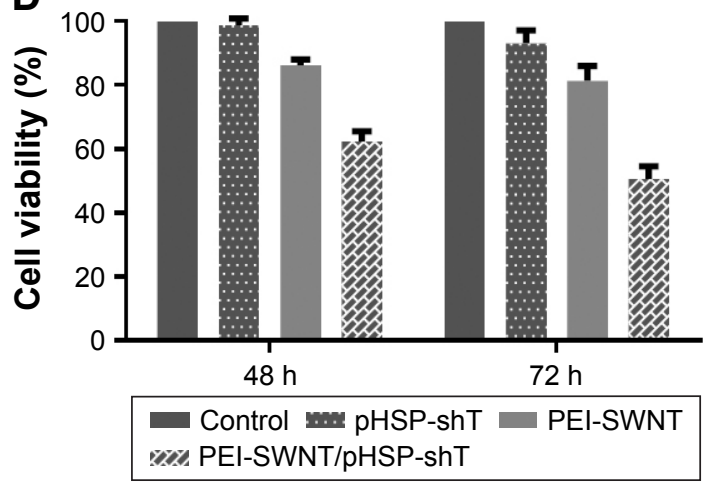

E
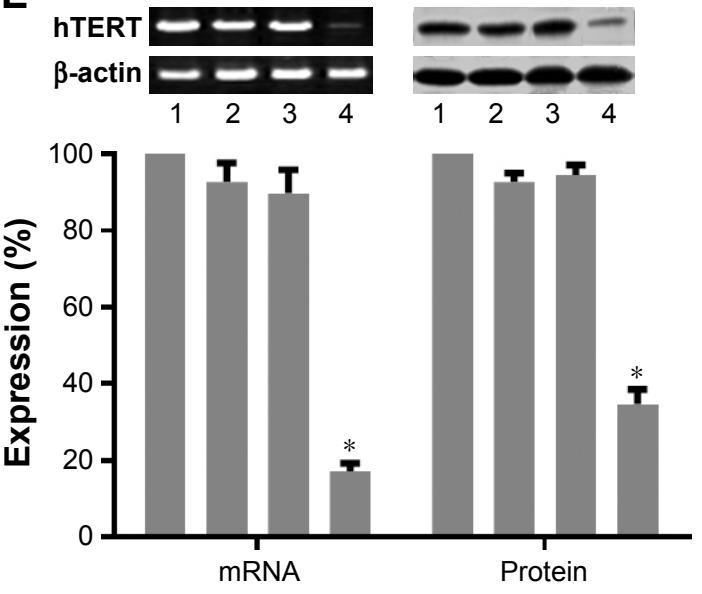

1 Control 2 pHSP-shT 3 PEI-SWNT $4 \mathrm{PEI}-\mathrm{SWNT} / \mathrm{pHSP}-\mathrm{shT}$

Figure 4 GFP expression and RNAi efficiency in MCF-7 cells. (A) The GFP expression plasmid pCMV-GFP was transfected into MCF-7 cells in the absence or presence of PEI-SWNT. (B) The Hsp70B' promoter-driven GFP expression plasmid pHSP-GFP was loaded onto PEI-SWNT and transfected into MCF-7 cells in the absence or presence of NIR $(808 \mathrm{~nm})$ irradiation. (C) The heat-inducible shRNA plasmid pHSP-shGFP targeting GFP was co-transfected into MCF-7 cells with the pCMV-GFP plasmid at a ratio of I:I, followed by conditional exposure of the transfected cells to $808 \mathrm{~nm} \mathrm{NIR}$. The scale bar is $50 \mu \mathrm{m}$. (D) Cell viability of MCF-7 cells transfected with pHSP-shT, PEI-SWNT, and PEI-SWNT/pHSP-shT at different times. (E) mRNA and protein expression of hTERT in MCF-7 cells. Transfections were conducted at $3 \mu \mathrm{g}$ plasmid and $0.5 \mu \mathrm{g}$ PEI-SWNT per well in six-well plates. Cells were treated with $808 \mathrm{~nm}$ laser irradiation for $45 \mathrm{~s}$. $* \mathrm{P}<0.0 \mathrm{I}$, PEI-SWNT/pHSP-shT versus the other three groups.

Abbreviations: GFP, green fluorescent protein; CMV, cytomegalovirus; HSP, heat-shock protein; NIR, near-infrared; hTERT, human telomerase reverse transcriptase; PEI-SWNT, polyetherimide-modified single-wall carbon nanotube; sh, short hairpin; shT, short hairpin RNA of human telomerase reverse transcriptase.

time-dependent manner. Moreover, significant cell apoptosis and tumor growth were observed under $808 \mathrm{~nm}$ laser radiation at $1.5 \mathrm{~W} / \mathrm{cm}^{2}$ for $45 \mathrm{~s}^{10}$ To evaluate the NIR-induced transcriptional activity of the Hsp70B' promoter, pHSP-GFP was loaded onto PEI-SWNT and transfected into MCF-7 cells. As shown in Figure 4B, we observed extensive GFP expression in PEI-SWNT/pHSP-GFP-transfected cells following exposure to $808 \mathrm{~nm}$ laser irradiation for $45 \mathrm{~s}$, whereas cells not exposed to laser irradiation showed no GFP expression. These results indicated that PEI-SWNT successfully transported the pHSP-GFP plasmid into MCF-7 cells and enabled transcription of the reported gene via the Hsp70B' promoter.

The heat-induced RNAi plasmid pHSP-shGFP was then used to estimate RNAi-specific effects triggered by NIR.
The indicated RNAi plasmid was co-transfected into MCF-7 cells with pCMV-GFP via PEI-SWNT. Upon exposure to laser irradiation, the Hsp70B' promoter was activated, and it generated shRNA targeting GFP, resulting in reduced GFP expression. By contrast, in the absence of laser irradiation, pCMV-GFP expressed GFP based on the absence of shRNA activity. As shown in Figure 4C, green fluorescence was diminished in the irradiated cells compared with the control cells, which confirmed the above inference. To further confirm that PEI-SWNT was responsible for heat-induced RNAi efficacy, pHSP-shGFP and pCMV-GFP were co-transfected into MCF-7 cells using ExGen500 instead of PEI-SWNT. As expected, we observed extensive green fluorescence in cells independent of laser irradiation (data not shown), suggesting that PEI-SWNT mediated NIR-responsive RNAi activity. 


\section{NIR-responsive antitumor activity in vitro}

Confirmation of the RNAi effect via the Hsp70B' promoter that could be triggered by NIR-specific activity associated with PEI-SWNT suggested that this novel stimulus-responsive nano-gene-delivery system could potentially be used in targeted therapy for breast cancer. Breast cancer is the most common malignant tumor among women, with incidence increasing with age over $35 .^{22}$ Studies have revealed that hTERT is a rate-limiting enzyme involved in telomerase activity and is specifically expressed in tumor cells as an essential factor in the initiation and development of breast cancer. ${ }^{23,24}$ Knockdown of hTERT expression results in dramatic suppression of breast cancer cell proliferation; ${ }^{25}$ therefore, hTERT was selected as a target gene for this study.

To detect the effectiveness of NIR-responsive gene therapy in vitro, a proven sequence inducing significant gene suppression on hTERT was used to construct the heat-induced RNAi plasmid pHSP-shT, which was transfected into MCF-7 cells via PEI-SWNT. As shown in Figure 4D, MTT results revealed that cell viability in the PEI-SWNT/pHSP-shTtransfected group significantly decreased following exposure to $808 \mathrm{~nm}$ laser irradiation for $45 \mathrm{~s}$. By contrast, no significant difference in cell viability was observed between the

A

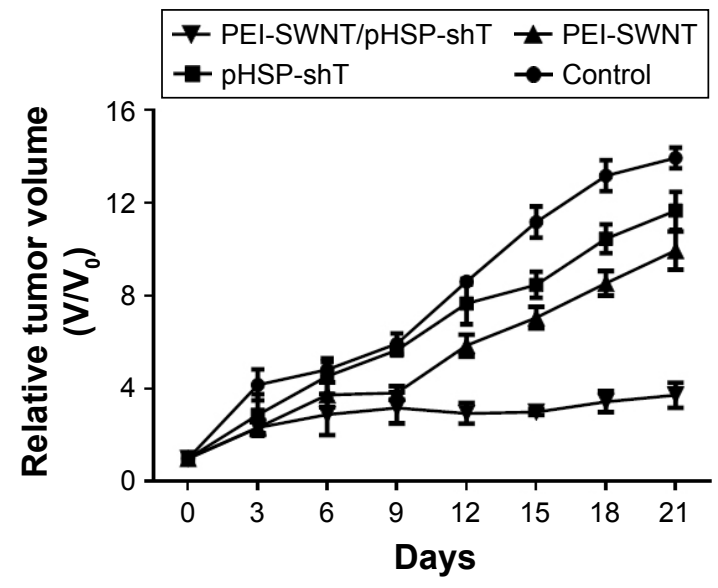

C
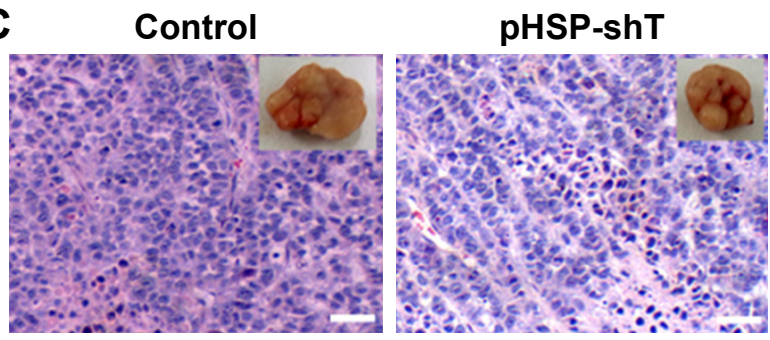

PEI-SWNT/pHSP-shT-transfected group and control groups in the absence of NIR laser irradiation (Figure S2).

Furthermore, we evaluated the effect of NIR-responsive hTERT knockdown at the mRNA and protein levels. As shown in Figure 4E, hTERT mRNA and target protein levels in cells transfected with PEI-SWNT/pHSP-shT decreased to $17.22 \% \pm 2.03 \%$ and $34.58 \% \pm 3.87 \%$, respectively, following $808 \mathrm{~nm}$ laser irradiation. These results confirmed that the PEI-SWNT/pHSP-shT nanoparticles were capable of inhibiting hTERT expression and tumor cell proliferation upon exposure to laser irradiation because of NIR-responsive RNAi-specific activity.

\section{NIR-responsive antitumor activity in vivo}

To investigate the in vivo antitumor activity of the PEISWNT/pHSP-shT nanoparticles in response to NIR, tumor models were established in nude mice that were subsequently divided into four groups (control, pHSP-shT, PEI-SWNT, and PEI-SWNT/pHSP-shT). We observed changes in tumor volume in each group, indicating increasing trends according to the period of administration (Figure 5A and S3). In the absence of laser irradiation, no significant changes were observed among the four groups (Figure S3); however,

\section{B}
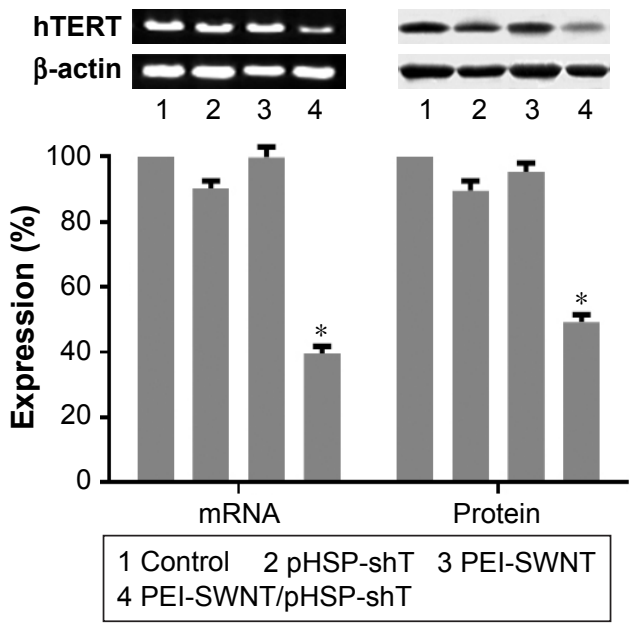

PEI-SWNT

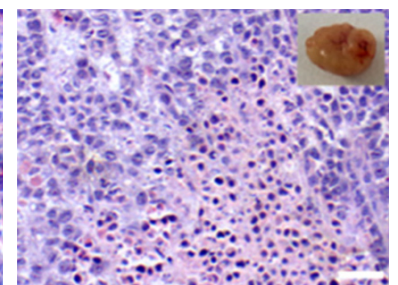

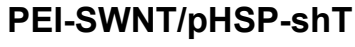

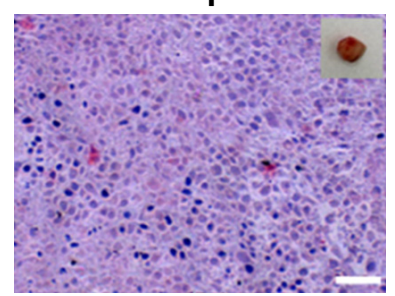

Figure 5 Antitumor activity in vivo. (A) Relative tumor volume in mice from different treatment groups. (B) hTERT mRNA and protein expression in MCF-7 tumor-bearing mice in vivo. $* P<0.0$ I, PEI-SWNT/pHSP-shT versus the other three groups. (C) H\&E-stained tumor tissues in mice. The scale bar is $50 \mu \mathrm{m}$.

Abbreviations: HSP, heat-shock protein; hTERT, human telomerase reverse transcriptase; PEI-SWNT, polyetherimide-modified single-wall carbon nanotube; shT, short hairpin RNA of human telomerase reverse transcriptase. 
following laser irradiation, tumor growth in the pHSP-shT group was similar to that observed in the control group, whereas that in the PEI-SWNT group was inhibited. Notably, there was also no significant difference between the saline control groups in the presence or absence of laser irradiation. This was consistent with previous results indicating that PEI-SWNT was essential for transforming NIR energy to thermal energy. In agreement with this finding, we observed that PEI-SWNT/pHSP-shT exhibited significant reduction in relative tumor volume relative to that observed in the control group, indicating the successful application of NIR to RNAi-based gene therapy to induce an antitumor effect in vivo.

We then tested NIR-responsive gene silencing by measuring hTERT mRNA and protein expression by RT-PCR and Western blot, respectively. Our results showed that hTERT expression in the PEI-SWNT/pHSP-shT group decreased significantly $(P<0.01)$, resulting in decreases in mRNA and protein levels by $60.24 \% \pm 2.12 \%$ and $50.66 \% \pm 2.24 \%$, respectively (Figure 5B). This result indicated that the PEISWNT/pHSP-shT nanoparticle was successfully activated by $808 \mathrm{~nm}$ NIR radiation, resulting in NIR-responsive RNAibased targeting of hTERT.

We confirmed tumor-specific damage via NIR-responsive gene silencing by H\&E staining. The pHSP-shT group showed no significant difference relative to the control group (Figure 5C), whereas tumor cell number in the PEI-SWNT group decreased, and the cell nucleus became pyknotic, likely due to the thermal energy generated by PEI-SWNT. Additionally, tumor cells in the PEI-SWNT/pHSP-shT group exhibited significant decreases in cell number, cell staining indicative of decreased viability, and random orientation, indicating that NIR-responsive gene silencing promoted antitumor activity.

\section{In vivo toxicity of the NIR-responsive gene silencing system}

Along with significant tumor inhibition, low toxicity is also an essential characteristic of an effective gene therapy system. One method used to measure toxicity in vivo involves monitoring weight fluctuations in the animal model, because high toxicity in vivo consistently results in weight loss. As shown in Figure 6A, the weight of the mice in each group continued to increase steadily, with no significant difference observed among groups $(P>0.05)$, suggesting that the PEISWNT/pHSP-shT nanoparticle might be atoxic.

Moreover, we used the visceral index and H\&E staining to determine in vivo toxicity. As shown in Figure 6B, the visceral indices of the heart, liver, spleen, lung, and kidney in each group did not differ significantly from those of the control group. Furthermore, H\&E staining (Figure 6C) showed that the control group exhibited no abnormal organ or cell morphology, with cells exhibiting normal groupings and clear boundaries. Similar results were also observed in other groups, confirming that the NIR-responsive gene silencing system achieved excellent tumor-specific targeting without sacrificing safety.

\section{Conclusion}

In this study, we report the effects of a PEI-SWNT/pHSPshT-based NIR-activatable RNAi system for breast cancer gene therapy. There are two essential elements in this NIRresponsive gene therapy system: one is a PEI-modified SWNT capable of transfecting RNAi vectors into tumor cells

B

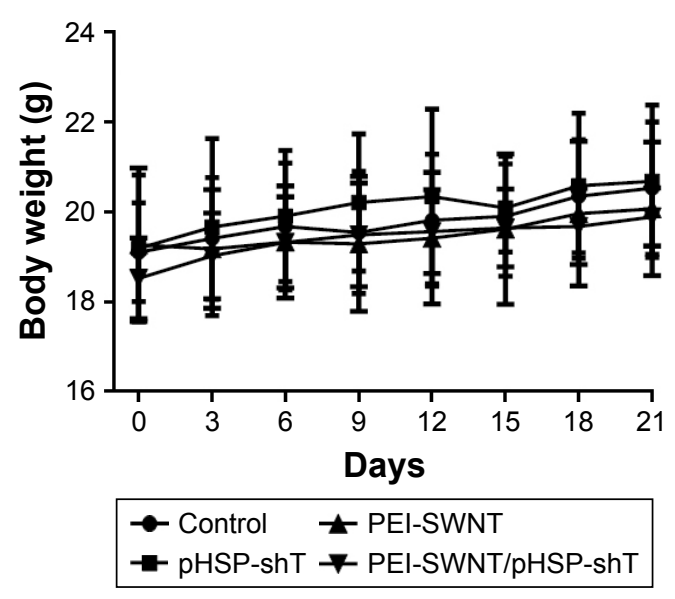

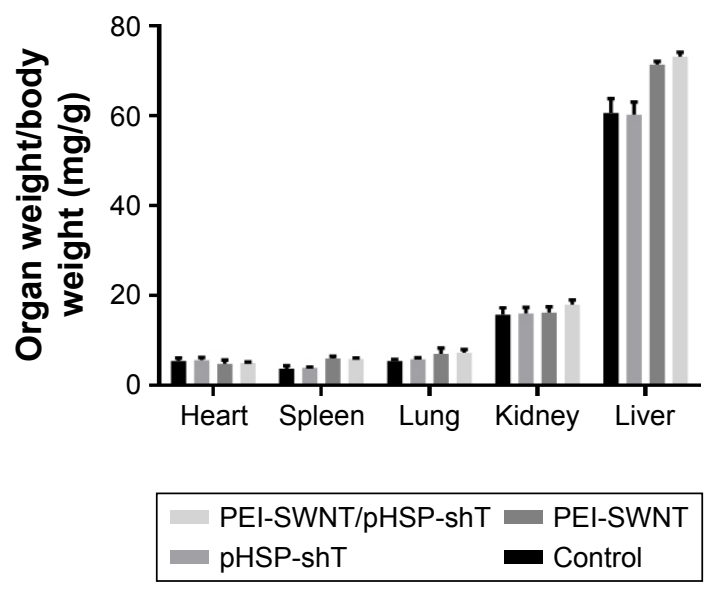

Figure 6 (Continued) 
C
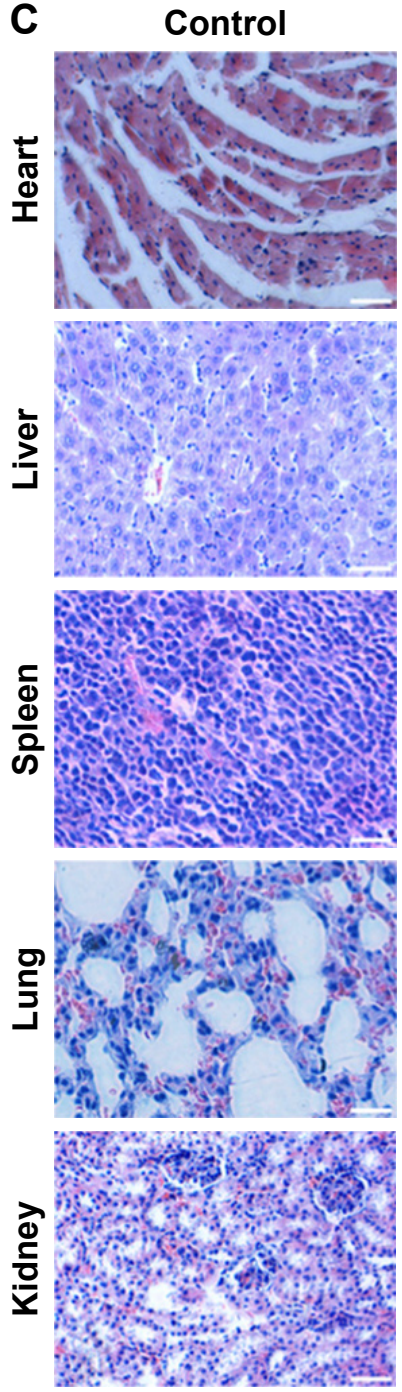

PEI-SWNT
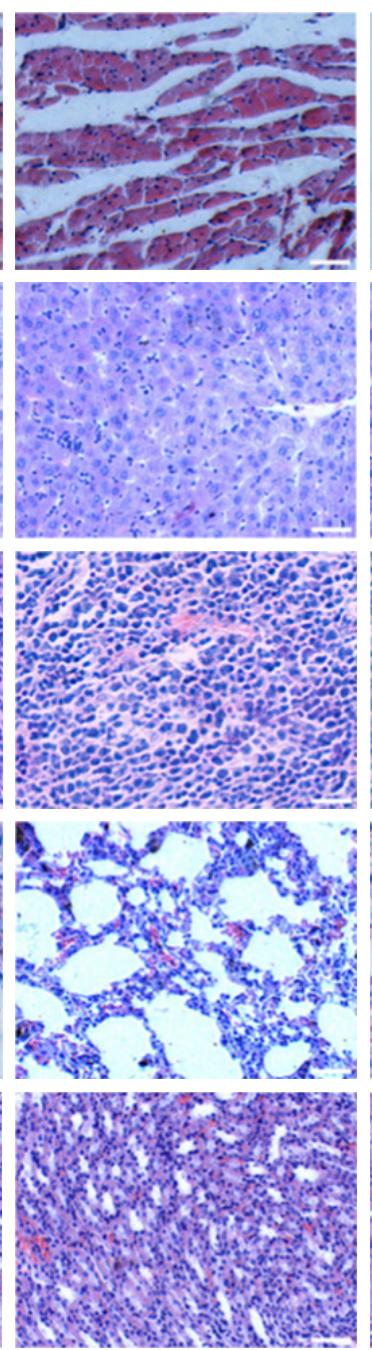

pHSP-shT
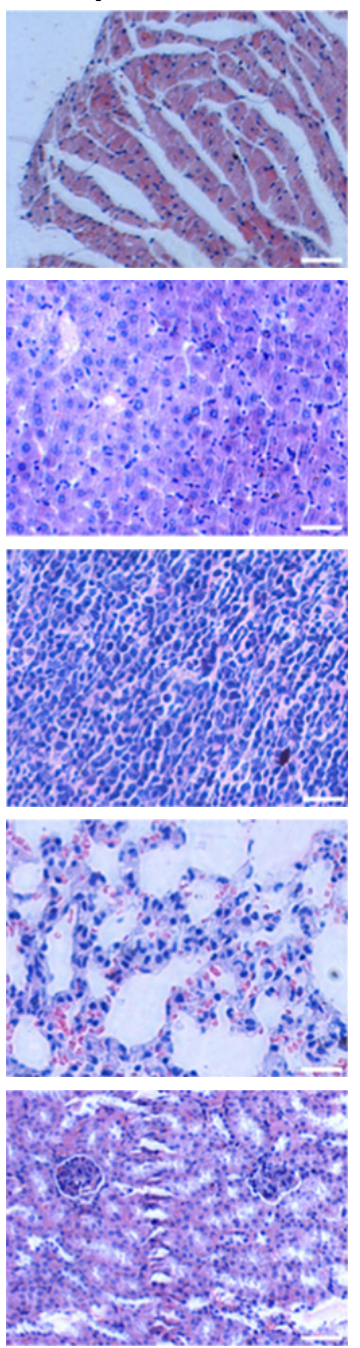

PEI-SWNT/pHSP-shT
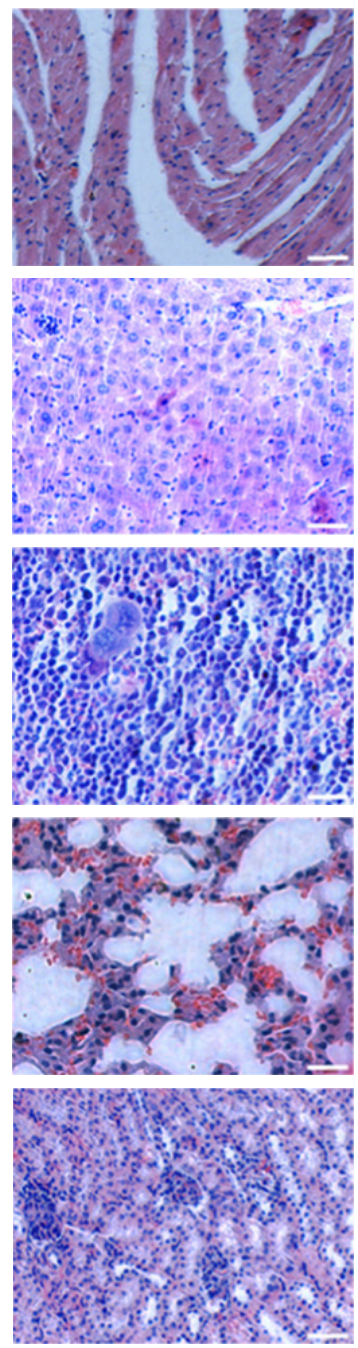

Figure 6 In vivo toxicity. Changes in (A) mouse body weight, and (B) organ weight. (C) H\&E stained major organs harvested from mice. The scale bar is $50 \mu$ m. Abbreviations: HSP, heat-shock protein; PEI-SWNT, polyetherimide-modified single-wall carbon nanotube; shT, short hairpin RNA of human telomerase reverse transcriptase.

and generating light-heat conversion upon irradiation, and the other is an Hsp70 $\mathrm{B}^{\prime}$ promoter capable of thermal control of targeted gene silence. Although the strategy established in this study made it possible to achieve controlled gene knockdown successfully, leading to enhancement of gene therapy effectiveness and mitigation of off-target effects, a PEI-SWNT/pHSP-shT based NIR-activatable RNAi system may be inherently limited by inhomogeneous photothermal effect and limited NIR penetration depth in the tumor. Moreover, it should not be neglected that the significant decrease of hTERT expression in vivo was similar to but less than that in vitro. The chief reason for the difference is the positive charge of PEI-SWNT/pHSP-shT, which hindered the systemic delivery of DNA to tumor cells by nonspecific interaction between nanoparticles and serum components in vivo. Therefore, our future studies will focus on the optimization of this stimulus-responsive RNAi system, which will contribute to new therapeutic strategies associated with cancer gene therapy.

\section{Acknowledgments}

This work was supported by the National Natural Science Foundation of China (grant no 81573364). The authors are grateful to Dr Jinjin Shi for technical assistance.

\section{Disclosure}

The authors report no conflicts of interest in this work.

\section{References}

1. Conde J, Ambrosome A, Hernandez Y, et al. 15 years on siRNA delivery: beyond the State-of-the-Art on inorganic nanoparticles for RNAi therapeutics. Nano Today. 2015;10(4):421-450. 
2. Guo J, Jiang X, Gui S. RNA interference-based nanosystems for inflammatory bowel disease therapy. Int J Nanomedicine. 2016;11: 5287-5310.

3. Ding C, Tong L, Feng J, Fu J. Recent advances in stimuli-responsive release function drug delivery systems for tumor treatment. Molecules. 2016;21(12):1715

4. Kim H, Chung K, Lee S, Kim DH, Lee H. Near-infrared light-responsive nanomaterials for cancer theranostics. Wiley Interdiscip Rev Nanomed Nanobiotechnol. 2016;8(1):23-45.

5. Li D, Ma Y, Du J, et al. Tumor acidity/NIR controlled interaction of transformable nanoparticle with biological systems for cancer therapy. Nano Lett. 2017;17(5):2871-2878.

6. Prabhakar N, Zhang J, Desai D, et al. Stimuli-responsive hybrid nanocarriers developed by controllable integration of hyperbranched PEI with mesoporous silica nanoparticles for sustained intracellular siRNA delivery. Int J Nanomedicine. 2016;11:6591-6608.

7. Chen P, Kanehira K, Sonezaki S, Taniguchi A. Detection of cellular response to titanium dioxide nanoparticle agglomerates by sensor cells using heat shock protein promoter. Biotechnol Bioeng. 2012;109(12): 3112-3118.

8. Noonan EJ, Place RF, Giardina C, Hightower LE. Hsp70B' regulation and function. Cell Stress Chaperones. 2007;12(3):219-229.

9. Andersson HA, Kim YS, O’Neill BE, Shi ZZ, Serda RE. HSP70 promoter-driven activation of gene expression for immunotherapy using gold nanorods and near infrared light. Vaccines (Basel). 2014;2(2): 216-227.

10. Wang L, Shi J, Zhang H, et al. Synergistic anticancer effect of RNAi and photothermal therapy mediated by functionalized single-walled carbon nanotubes. Biomaterials. 2013;34(1):262-274.

11. Lin J, Liu Y, Zhan Y, et al. Synthetic Tet-inducible small hairpin RNAs targeting hTERT or Bcl-2 inhibit malignant phenotypes of bladder cancer T24 and 5637 cells. Tumor Biol. 2016;37(3):3115-3121.

12. Leung TK, Rajendran MY, Monfries C, Hall C, Lim L. The human heat-shock protein family. Expression of a novel heat-inducible HSP70 (HSP70B') and isolation of its cDNA and genomic DNA. Biochem J. 1990;267(1):125-132.

13. Liao FH, Shui JW, Hsing EW, et al. Protein phosphatase 4 is an essential positive regulator for Treg development, function, and protective gut immunity. Cell Biosci. 2014;4:25.
14. Shi J, Chen Z, Wang L, et al. A tumor-specific cleavable nanosystem of PEG-modified C60@Au hybrid aggregates for radio frequencycontrolled release, hyperthermia, photodynamic therapy and X-ray imaging. Acta Biomater. 2016;29:282-297.

15. Shinagawa T, Ishii S. Generation of Ski-knockdown mice by expressing a long double-strand RNA from an RNA polymerase II promoter. Genes Dev. 2003;17(11):1340-1345.

16. Ren X, Ma Y, Xu M, Chen T, Zhang Z, Zhang Y. Construction, modification and evaluation of apolipoprotein A-I promoter-driven shRNA expression vectors against hTERT. Plasmid. 2011;65(1):42-50.

17. Mirzaei H, Sahebkar A, Jaafari MR, et al. PiggyBac as a novel vector in cancer gene therapy: current perspective. Cancer Gene Ther. 2016; $23(2-3): 45-47$.

18. Ibraheem D, Elaissari A, Fessi H. Gene therapy and DNA delivery systems. Int J Pharm. 2014;459(1-2):70-83.

19. Li Z, de Barros ALB, Soares DCF, Moss SN, Alisaraie L. Functionalized single-walled carbon nanotubes: cellular uptake, biodistribution and applications in drug delivery. Int J Pharm. 2017;524(1-2):41-54.

20. Neves LF, Krais JJ, Van Rite BD, Ramesh R, Resasco DE, Harrison RG Targeting single-walled carbon nanotubes for the treatment of breast cancer using photothermal therapy. Nanotechnology. 2013;24(37) 375104.

21. Han S, Kwon T, Um JE, Haam S, Kim WJ. Highly selective photothermal therapy by a phenoxylated-dextran-functionalized smart carbon nanotube platform. Adv Healthc Mater. 2016;5(10):1147-1156.

22. Lee GE, Mayer EL, Partridge A. Prognosis of pregnancy-associated breast cancer. Breast Cancer Res Treat. 2017;163(3):417-421.

23. Hashemi M, Amininia S, Ebrahimi M, Hashemi SM, Taheri M, Ghavami S. Association between hTERT polymorphisms and the risk of breast cancer in a sample of Southeast Iranian population. BMC Res Notes. 2014;7:895.

24. Dwyer JM, Liu JP. Ets2 transcription factor, telomerase activity and breast cancer. Clin Exp Pharmacol Physiol. 2010;37(1):83-87.

25. Rao Y, Xiong W, Liu H, et al. Inhibition of telomerase activity by dominant-negative hTERT retards the growth of breast cancer cells Breast Cancer. 2016;23(2):216-223. 


\section{Supplementary materials}
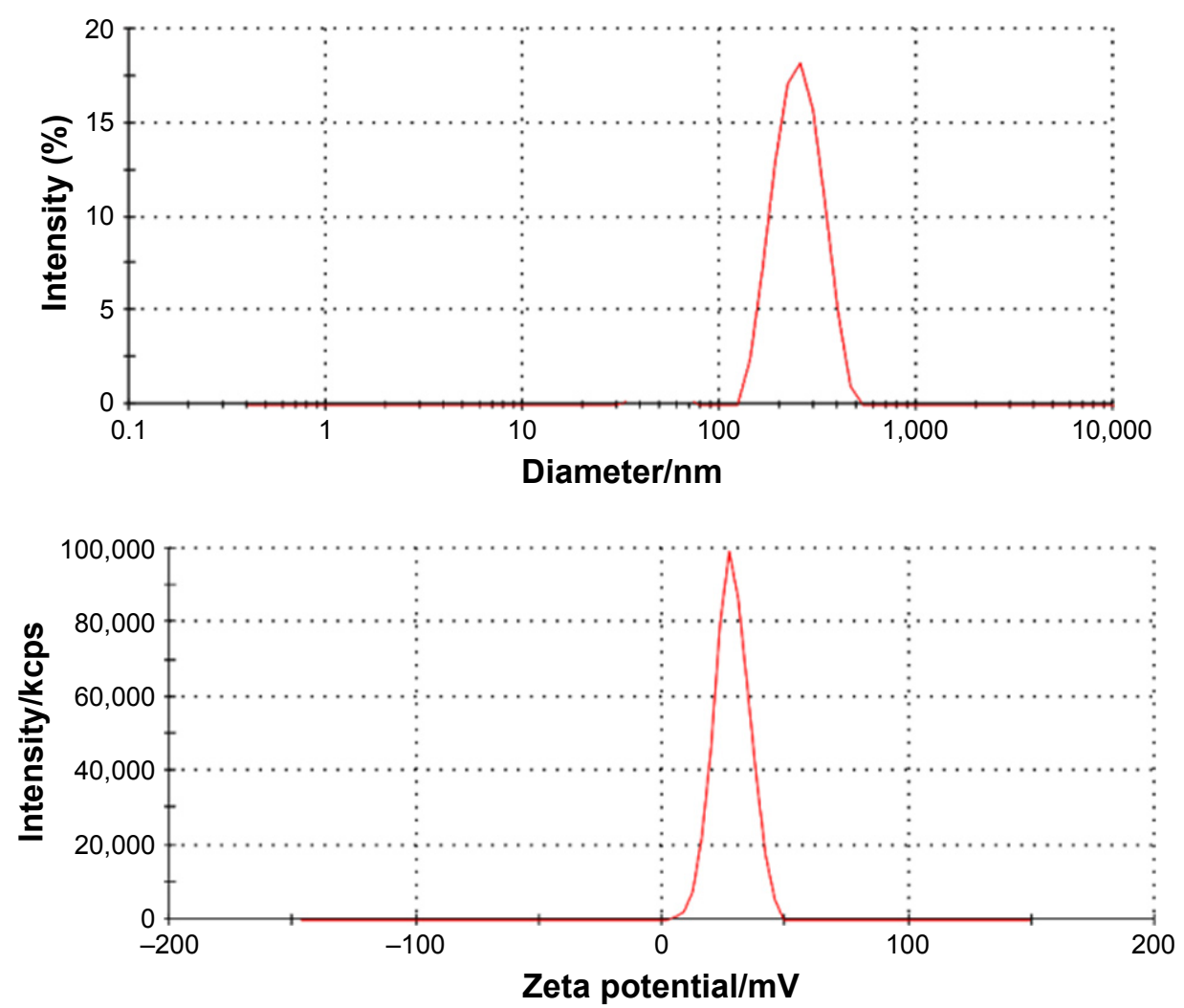

Figure SI The size and zeta-potential of PEI-SWNT.

Abbreviation: PEI-SWNT, polyetherimide-modified single-wall carbon nanotube.

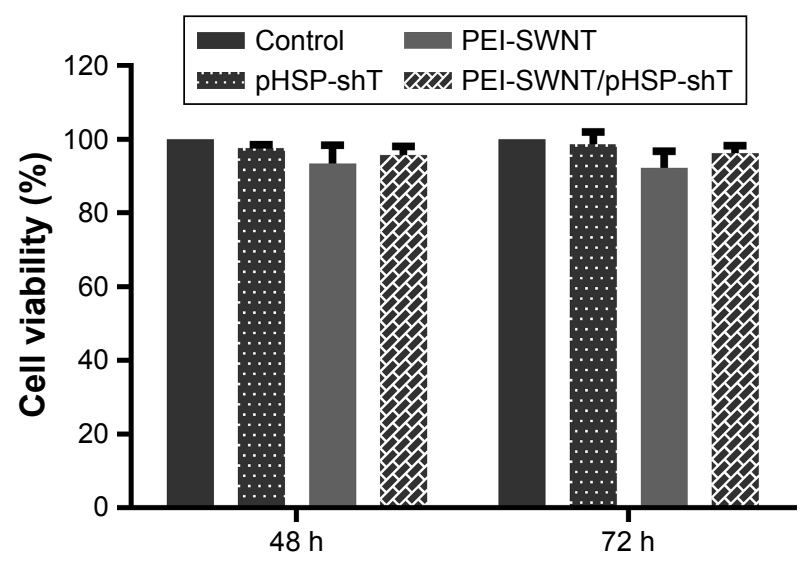

Figure S2 Cell viability of MCF-7 cells transfected with pHSP-shT, PEI-SWNT, and PEI-SWNT/pHSP-shT in the absence of NIR laser irradiation.

Abbreviations: HSP, heat-shock protein; PEI-SWNT, polyetherimide-modified single-wall carbon nanotube; shT, short hairpin RNA of human telomerase reverse transcriptase.

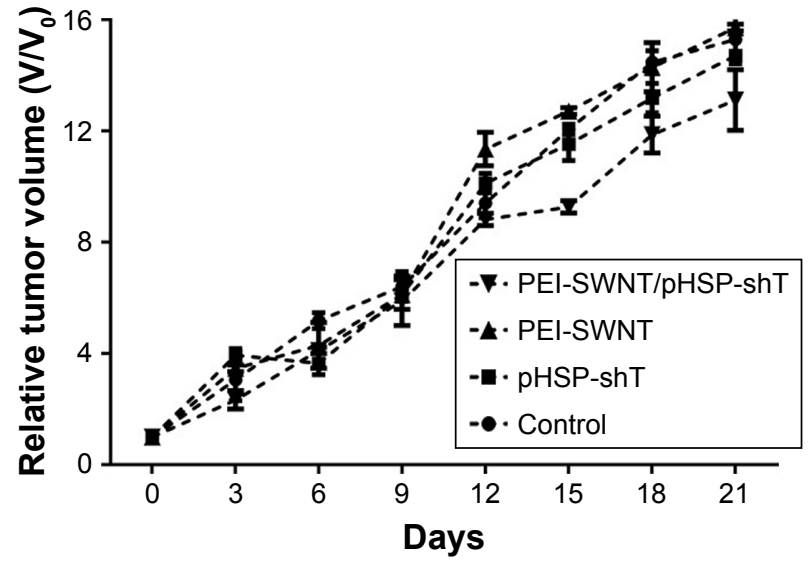

Figure S3 Relative tumor volume of the four groups in the absence of laser irradiation.

Abbreviations: HSP, heat-shock protein; PEI-SWNT, polyetherimide-modified single-wall carbon nanotube; shT, short hairpin RNA of human telomerase reverse transcriptase.
International Journal of Nanomedicine

\section{Publish your work in this journal}

The International Journal of Nanomedicine is an international, peerreviewed journal focusing on the application of nanotechnology in diagnostics, therapeutics, and drug delivery systems throughout the biomedical field. This journal is indexed on PubMed Central, MedLine, CAS, SciSearch ${ }^{\circledR}$, Current Contents ${ }^{\circledR} /$ Clinical Medicine,

\section{Dovepress}

Journal Citation Reports/Science Edition, EMBase, Scopus and the Elsevier Bibliographic databases. The manuscript management system is completely online and includes a very quick and fair peer-review system, which is all easy to use. Visit http://www.dovepress.com/ testimonials.php to read real quotes from published authors. 